\title{
INVESTIGATING ISSUES OF DISPLAY CONTENT VS. CLUTTER DURING AIR-TO-GROUND TARGETING MISSIONS
}

\author{
Maura C. Lohrenz \\ Naval Research Laboratory Code 7440.1 \\ Stennis Space Center, MS 39529 \\ R. John Hansman \\ International Center for Air Transportation \\ Massachusetts Institute of Technology \\ Cambridge, MA 02139
}

\begin{abstract}
This paper investigates competing influences of display content and clutter on pilot performance during flight guidance and target acquisition phases of air-to-ground targeting missions. Based on interviews with F/A-18 pilots, a cognitive process model is presented to help understand how pilots use and transition between internal and external sources of information to support decision-making and aircraft control. Experiments were conducted in which subjects flew targeting missions using a flight simulator connected to the Navy's FalconView moving-map. In one experiment, subjects referred to three versions of the display: 1) a detailed map overlaid with critical mission symbology, 2) the map only, and 3) mission overlays only. Flight guidance and target acquisition performances were best with the overlays-only display. Performance was comparable or worse with the combination display and significantly worse with the map only, suggesting that the distraction of map clutter countered the benefits of map content.
\end{abstract}

\section{INTRODUCTION}

A critical issue in military aviation is the pilot's ability to transition between internal (head-down, instrument-driven) and external (head-up, out of the cockpit) guidance. Military pilots interviewed for this project reported difficulties during this transition, particularly during time-critical, air-to-ground missions such as Close Air Support (CAS) and Forward Air Control (FAC).

Air-to-ground missions require precise flight guidance to reach the target area and timely, accurate target acquisition to search for, locate, and identify the target. These mission phases are interdependent: errors during flight guidance may result in failure to acquire the target; conversely, flight guidance is a series of target acquisitions as a pilot searches for "lead-in" features along the route (Conejo and Wickens, 1997). Flight guidance is supported by a map or electronic moving-map display (internal guidance cues), while target acquisition relies on intelligence - often communicated by troops on the ground (e.g., FAC-G) or other aircraft (FAC-A) - and focuses on external cues. The pilot must correlate internal and external guidance cues throughout the mission - a process termed navigational checking (Wickens and Long, 1994) - with the focus on external cues commanding more attention as the target area approaches.

The Navy has used the cockpit moving-map display as a situational awareness tool for over a decade. Current systems display raster images, such as scanned charts and satellite imagery. Future systems will use vector databases in which individual map features can be manipulated, allowing the display to be customized and queried (Lohrenz, et al., 1997). As new data becomes available, there is a tendency to present everything that might be of interest. These new displays introduce human factors issues concerning the pilot's ability to access and interpret the displayed information. Many studies have linked display complexity to pilot performance (e.g.,
Aretz, 1988). Display "clutter" - including unwanted noise and overcrowding of otherwise important information - is thought to disrupt a pilot's visual attention, resulting in greater uncertainty about target locations. A premise of this paper is that display clutter can hinder a pilot's ability to correlate internal and external guidance cues.

Three F/A-18 pilots were interviewed to discuss how the moving-map display supports their missions and how it could be improved (Lohrenz, 2003). The pilots identified the moving-map as one of several channels of information they use to make decisions during time-critical air-to-ground missions. Figure 1 presents a cognitive process model developed from these interviews to help visualize how pilots integrate the often-disparate sources of information to support decision-making and aircraft control. The model includes 1) the pilot's preconceived mental model of the mission; 2) a 2D internal visual channel (moving-map, flight instruments); 3) a 3D external visual channel (real-world) supplemented by a head-up display (HUD); and 4) an aural channel for radio communications (e.g., with a FAC). The proposed model reinforces the importance of a pilot's ability to transition between internal and external guidance. Clutter in any of these information channels is expected to hinder the pilot's ability to correlate cues, impeding mission success.

The next sections present an experiment to investigate competing influences of display content and clutter on a pilot's ability to correlate internal and external cues, measured by subjects' performance and workload during flight guidance and target acquisition phases of a simulated air-to-ground targeting mission using three moving-map displays (figure 2):

a. Combination display: a topographic map with graphic overlays of flight path and target location,

b. Map-only display: the topographic map with no overlays, and

c. Overlays-only display: the mission-specific graphic overlays with no underlying map. 


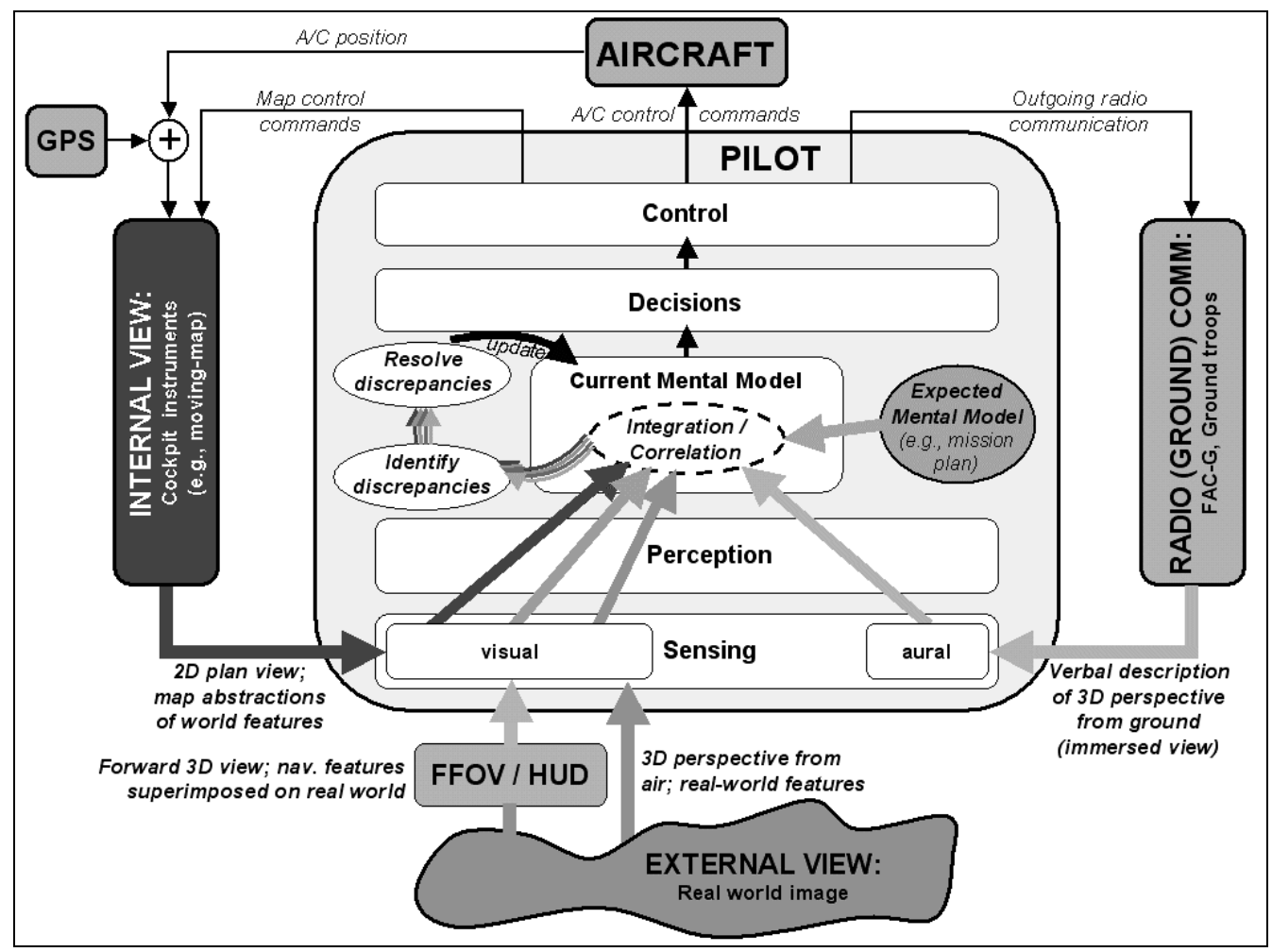

Figure 1. Cognitive process model of pilot integrating various channels of information to support decision-making and aircraft control.

a)

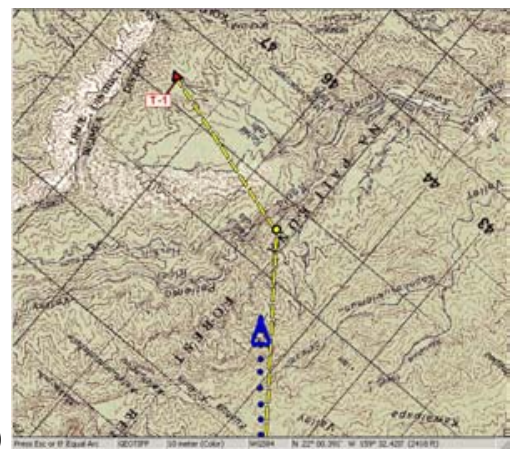

b)

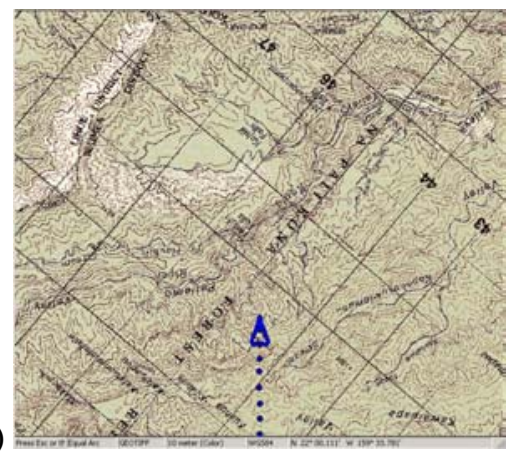

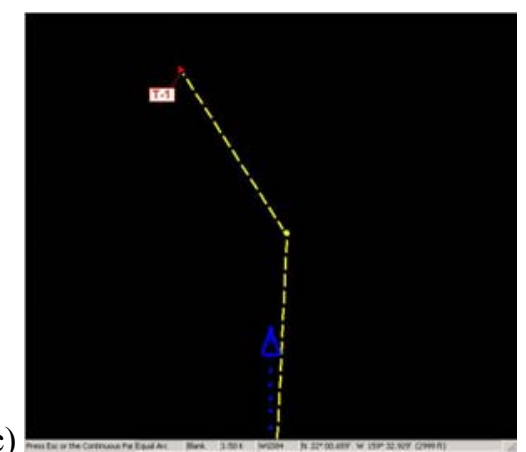

Figure 2. Three experimental display cases: a) combination; b) map only; and c) overlays only.

\section{METHODS}

Twelve volunteers (nine male, three female) were recruited from the Naval Research Laboratory Mapping, Charting and Geodesy Branch. Two participants held a pilots' license and three had flight simulator experience. Participants ranged in age from 24 to 52, with a mean age of 37.8 .

A laptop running Microsoft Flight Simulator 2002 (FS2002) generated flight scenes. Participants controlled pitch, roll, and throttle with a joystick. To maximize the simulator's outside view, the instrument panel was not displayed. Instead, a digital readout of latitude, longitude, altitude, heading, and airspeed was provided at the top of the display, along with an analog compass. The simulator scene was set for afternoon in Waimea Canyon, Kauai, with unlimited visibility, no precipitation and no winds. Since most subjects had little to no flying experience, the aircraft was set to the relatively stable, easily controlled Cessna 172 .

A second laptop running the Navy's FalconView program generated a moving-map. The two laptops were linked via serial cable, and software was written to send position information at a rate of $1 \mathrm{~Hz}$ from FS2002 to FalconView, such that the moving-map was controlled by FS2002. For the map-only and combination displays, a 1:25,500 scale topographic chart of Kauai was displayed track-up. For all three displays, a triangular icon represented the aircraft's position, and a "breadcrumb trail" indicated where the aircraft had already flown.

Each subject flew one mission per test case. For each mission, subjects were given two primary flight guidance 
tasks: stay alive (i.e., don't crash) and stay on the planned route. Subjects' primary target acquisition tasks were to stay alive and to find and identify a target (described below) as quickly as possible, following instructions given $2 \mathrm{~km}$ from the target. Subjects were told there would be one real target and one false target on each mission. They were to search for and say the name of the real target (e.g., "tank") as soon as possible and simultaneously type "o" on the laptop. The "o" was recorded to time the subject's target acquisition task (from the time at which target instructions were given). If the subject identified the correct target, the mission was complete. If not, the subject was told to keep searching. Secondary tasks for both phases were to maintain altitude (specified at mission start) and airspeed (between 80-160 knots).

One of three real targets was included in each scene: a tank, jeep, or fuel truck. A car was included as the false target. All four vehicles were of similar size and camouflage color, so subjects had to scan the scene carefully to find the real target. Three route/target pairs were prepared to minimize subjects' familiarity with the routes and targets. The fuel truck was the target for route 1 , the jeep for route 2 , and the tank for route 3 . However, subjects were told that any target might be found on any route. Prior to starting the experiment, subjects flew a training mission that included all three targets plus the false target. Subjects could repeat this training as often as they wanted before starting the experiment.

Subjects were debriefed after each mission (including training). They rated their workload for each phase from 1 (easy) to 10 (impossible), based on a modified Bedford scale (Roscoe and Ellis, 1990). Next, subjects prioritized the cues they relied on to accomplish each phase. Valid cues included the outside view, instrument readings, topographic map (if shown), mission overlays (if shown), and FAC instructions (provided by the experimenter). Subjects were encouraged to take a break between missions, if needed. Most completed the experiment in one sitting, which lasted approximately $35 \mathrm{~min}$.

Independent variables for this experiment were subject, display type, and route/target pair. Dependent variables were flight guidance performance (FP), target acquisition performance (TP), flight guidance workload (FW), and target acquisition workload (TW), calculated as follows. Time and aircraft position (latitude, longitude, altitude) were recorded by FS2002 once per second. The first 20 seconds of each flight were not recorded, to give subjects time to adjust to the simulator settings for that mission. FP was calculated as the root mean squared error (RMSE) between planned and actual flight paths during a mission's flight guidance phase. TP was the time required to find and correctly identify the target. FW and TW were subjectively assessed using the Bedford scale.

\section{HYPOTHESES}

1. The map-only display will result in the worst FP and TP, and highest FW and TW, because it lacks flight path and target location information, which are expected to be more important than the map.

2a. If map clutter is a significant issue, the overlays-only will result in the best FP and TP, and lowest FW and TW, since it provides critical mission cues without map clutter.
2b.If map clutter is not a significant issue, the combination display will result in comparable or better FP, TP, FW and TW, compared with the overlays-only display.

3. Subjects will primarily use internal visual guidance cues during flight guidance, and external visual cues and aural cues (FAC instructions) during target acquisition.

\section{RESULTS}

Figure 3 summarizes results for both mission phases.

\section{Flight guidance}

A least squares analysis identifies display type as a main effect on FP $(F(2,20)=14.71, p<0.001)$. As expected, the map-only display resulted in the worst FP: average RMSE for the map-only was more than twice that of the combination, a significant difference $(t=2.54, p<0.05)$. Average FP for maponly was also significantly worse than for overlays-only $(t=2.24, p<0.05)$. FP was equivalent for combination and overlays-only. Subjects reported no significant differences in FW, considered "tolerable" for all cases, on average.

\section{Target acquisition}

Two subjects could not find the target. Since it was impossible to measure time-to-target when the target was not found, and since neither display type nor route/target was common to the two cases, these subjects were considered outliers and removed from the population before examining TP. Least squares analysis indicates display type was the only main effect $(F(2,16)=4.05, p<0.05)$ for TP. T-tests show that TP with the map-only display was significantly worse than with overlays-only $(t=2.24, p<0.05)$, as expected, but only somewhat worse than with the combination $(t=1.65, p \cong 0.1)$. Average TP with the combination display was somewhat worse than with overlays-only, but this difference was not significant $(t=1.45, p>0.1)$. A T-test revealed a significant difference in average TW ratings between the map-only and combination displays $(t=1.92, p<0.1)$, suggesting that subjects thought they were working harder with the map-only, as expected. No other differences in TW were significant.

\section{Use of internal and external guidance cues}

Figure 4 summarizes the guidance cues subjects reported using during each mission phase, by display. For all three displays, most subjects relied more on internal cues (movingmap and instrument readings) during flight guidance than during target acquisition. With the map-only display, most subjects referred to the instrument readings for internal cues; with overlays-only and combination displays, most subjects referred to the moving-map for internal cues. With all three displays, subjects relied much more on external cues and FAC instructions during target acquisition than during flight guidance. With combination and overlays-only (but not maponly), most subjects continued attending to the map during target acquisition, suggesting that the overlay information supported the transition between internal and external cues. 


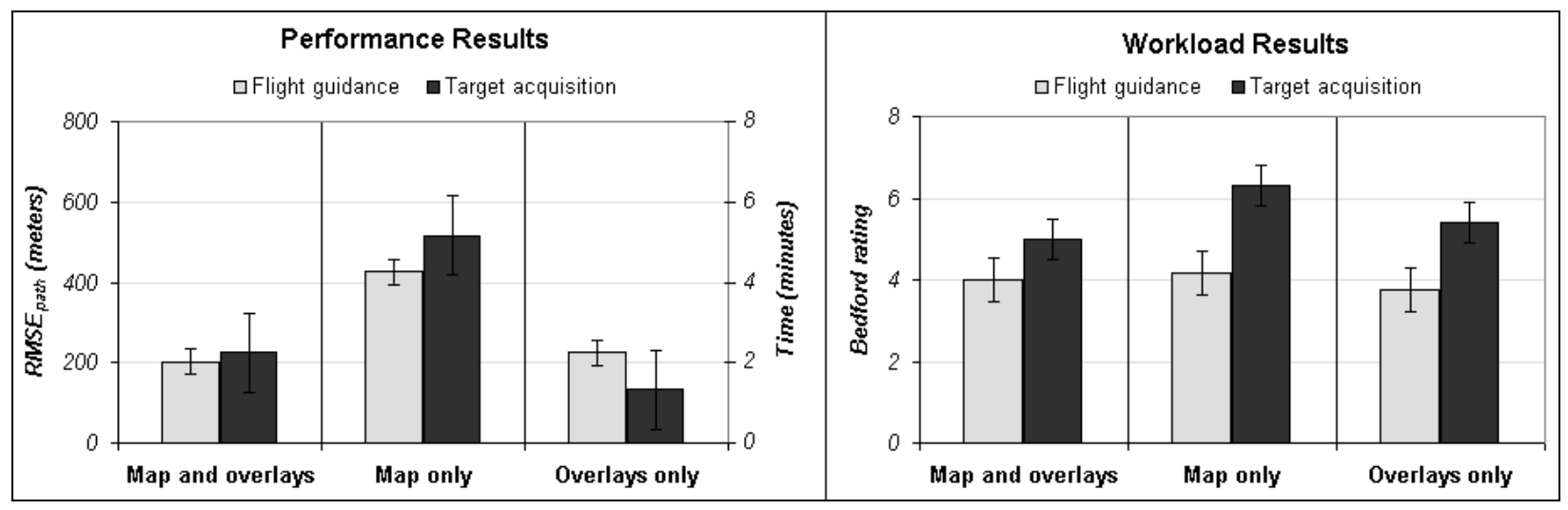

Figure 3. Summary of performance and workload results, by mission phase and display type.

Error bars represent one standard error above / below the mean.

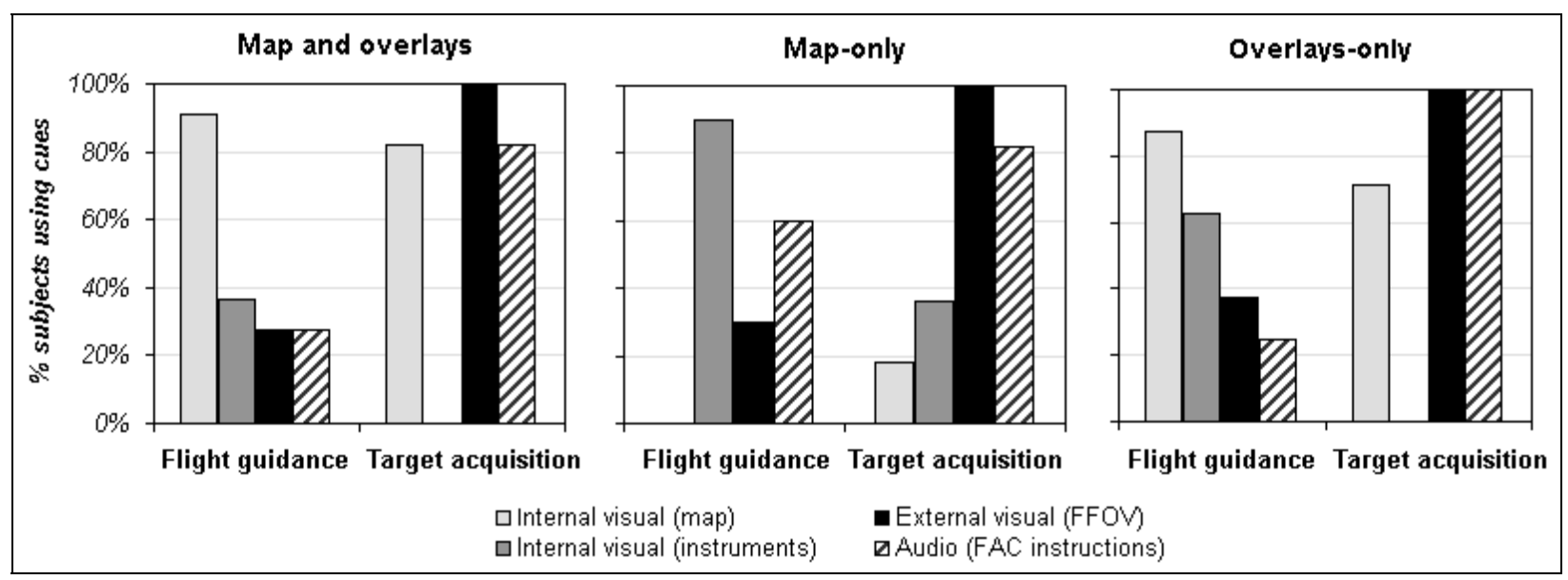

Figure 4. Percentage of subjects reporting use of four guidance cues, by mission phase and display type.

\section{DISCUSSION}

Results support the first hypothesis: the map-only display resulted in the poorest performance and highest workload for both flight guidance and target acquisition, suggesting that the map itself was less important than the mission-specific overlays in supporting the simulated air-to-ground mission.

Flight guidance results support hypothesis $2 \mathrm{~b}$ : map clutter did not appear to be a significant issue during this phase, since the combination display did not result in degraded performance or increased workload, compared with the overlays-only display.

Conversely, target acquisition results loosely support hypothesis 2a: map clutter may have been an issue during this phase, as evidenced by the combination display resulting in somewhat poorer performance than the overlays-only display, although workload was comparable.

Guidance cue results support the third hypothesis: most subjects reported using primarily internal visual guidance cues (map and/or instrument displays) during flight guidance, and primarily external visual and aural (FAC) cues during target acquisition. It is noteworthy that subjects also reported using the moving-map display during the target acquisition phase when overlays were displayed (i.e., combination and overlaysonly displays), but not when overlays were omitted (i.e., maponly display).

In summary, mission-specific overlays on the movingmap display provided more important internal visual cues than the underlying map for both flight guidance and target acquisition. If the overlays were present (with or without the map), performance was best, presumably because pilots had the necessary internal guidance cues to supplement their external scene. If the overlays were missing, pilots ignored the map display, attended mostly to external and aural cues, and exhibited poorer performance in both phases.

These results suggest that display designers should identify and present only the information required to accomplish a given task. Intelligent prioritization of information for display will become increasingly important as the number of potential sources of information increases. Different missions (and mission phases) often require different information to support the tasks to be performed, but presenting too much information at one time can degrade performance. Displays should provide a "declutter" interface to assist pilots in optimizing the display. Research is still needed to determine how best to perform this decluttering. 


\section{ACKNOWLEDGEMENTS}

This work was sponsored by the Naval Research Laboratory Select Graduate Training program and the Naval Air Systems Team (NAVAIR) Tactical Aircraft Moving Map Capabilities (TAMMAC) program. The authors thank Dan Shannon (TAMMAC deputy program manager) for his support. We thank the F/A-18 pilots at Pax River, MD, who shared their experiences with us, and the Naval Research Laboratory employees who participated in the experiment.

\section{REFERENCES}

Aretz, A. (1988). A model of electronic map interpretation. Proceedings of the Human Factors Society $32^{\text {nd }}$ Annual Meeting, Santa Monica, CA, pp. 130-135.

Conejo, R. and C.D. Wickens (1997). The effects of highlighting validity and feature type on air-to-ground target acquisition performance. Aviation Research Lab Technical Report ARL-97-11 / NAWC-ONR-97-1, University of Illinois at Urbana-Champaign. August.
Lohrenz, M.C. (2003). Cognitive issues related to advanced cockpit displays: supporting the transition between internal and external guidance. Thesis submitted in partial fulfillment of the requirements for the degree of Master of Science in Aeronautics and Astronautics at the Massachusetts Institute of Technology. June.

Lohrenz, M., M. Trenchard, S. Myrick, P. Van Zuyle, S. Fechtig (1997). Optimizing cockpit moving map displays for enhanced situational awareness. Chapter $13 \underline{\text { in }}$ Situational awareness in the tactical air environment: augmented proceedings of the Naval Air Warfare Center's first annual symposium, 363-387. CSERIAC, WrightPatterson AFB, OH. July.

Roscoe, A.H. and G.A. Ellis (1990). A subjective rating scale for assessing pilot workload in flight: a decade of practical use. Research Assessment Exercise (RAE) Technical Report TR90019.

Wickens, C. and J. Long (1994). Conformal symbology, attention shifts, and the head-up display. Proceedings of the 38th Annual Meeting of the Human Factors and Ergonomics Society, 6-10. Santa Monica, CA: Human Factors and Ergonomics Society. 